\title{
THE CHARACTER TABLE OF A MAXIMAL SUBGROUP OF THE MONSTER
}

\author{
R. W. BARRACLOUGH AND R. A. WILSON
}

\begin{abstract}
We calculate the character table of the maximal subgroup of the Monster $N(3 B) \cong 3_{+}^{1+12} \cdot 2 \cdot$ Suz:2, and also of the group $3^{1+12}: 6$. Suz:2, which has the former as a quotient. The strategy is to induce characters from the inertia groups in $3^{1+12}: 6$. Suz:2 of characters of $3^{1+12}$. We obtain the quotient map to $N(3 B)$ computationally, and our careful concrete approach allows us to produce class fusions between our tables and various tables in the GAP library.
\end{abstract}

\section{Introduction}

The large degree of the smallest faithful irreducible representation of $\mathbb{M}$ (196882 over $\mathbb{F}_{2}$ or $\mathbb{F}_{3}$ ) continues to make direct computation very difficult. All of the various constructions make heavy use of a maximal subgroup $[7,4,15,9]$, and computation in maximal subgroups continues to be an effective strategy for working in the Monster $[8,10,1]$.

The maximal subgroup $N(3 B) \cong 3_{+}^{1+12} \cdot 2 \cdot$ Suz:2 of the Monster continues to receive attention $[\mathbf{1 5}, \mathbf{1}]$, but the character table is unknown. Some of the other 'interesting' maximal subgroups of $\mathbb{M}$ have their character table stored in the GAP [6] character table library [3]; the most recent addition is $N(2 B) \cong 2^{1+24} \cdot \mathrm{Co}_{1}$; see [18]. Others are small enough for us to be able to compute the character table on demand. The subgroup $N(3 B)$ falls into neither category, so we aim to fill this irritating gap in our knowledge.

To make our tables useful they must integrate well into the GAP library. This means that we must produce fusion maps between known tables and our own tables, and name our conjugacy classes correctly.

\subsection{General strategy}

It is well known (see, for example, [11]) that the irreducible characters of a group of shape N.G can be obtained by inducing characters from the subgroups $N . T_{i}$ where each $T_{i}$ is the inertia group in $G$ of an irreducible character of $N$ (one $T_{i}$ for each orbit of $G$ on $\left.\operatorname{Irr} N\right)$. This is how we shall proceed.

Matters are complicated because our extension $3^{1+12} \cdot 2 \cdot \mathrm{Suz}: 2$ is non-split and there are two isomorphism classes of groups of this shape, only one of which is a subgroup of $\mathbb{M}$. We overcome the various difficulties by working in the larger group

The authors would like to thank John N. Bray for his helpful suggestions and discussions. Calculations were performed on equipment provided by EPSRC grant GR/R95265/01. The first author is supported by EPSRC grant GR/S41319/02.

Received 26 April 2006, revised 24 January 2007; published 22 May 2007.

2000 Mathematics Subject Classification 20C15, 20C45

(c) 2007, R. W. Barraclough and R. A. Wilson 
$3^{1+12}: 6 \cdot$ Suz:2 and use information from the computer construction of $\mathbb{M}[\mathbf{1 5}]$ to identify the correct quotient.

The inertia groups of $3^{1+12}: 6$. Suz: 2 acting on $\operatorname{Irr} 3^{1+12}$ are as follows.

1. $3^{1+12}: 6$ Suz:2. This case corresponds to the trivial character of $3^{1+12}$. The characters obtained are simply inflated from the quotient $6 \cdot$ Suz:2.

2. $3^{1+12}: 6$. Suz fixing the degree 729 characters of $3^{1+12}$. Inducing characters from this subgroup is easy once we have determined the values of the degree 729 characters extended to the inertia group, and the class fusion from $6 \cdot$ Suz to 6.Suz:2. This is the subject of Section 5.1.

3. $3^{1+12}:\left(\left(3 \times \mathrm{U}_{5}(2)\right): 2\right)$ fixes a linear character in an orbit of length 65520 . Induction from this subgroup is the subject of Section 6 .

4. $3^{1+12}:\left(3 \cdot 3^{5}:\left(\mathrm{M}_{11} \times 2\right)\right)$ fixes a linear character in an orbit of length 465920 . The induction is similar to case 3 . It is harder due to the larger index of the inertia group, but different module structure makes some parts of the calculation easier.

\section{The conjugacy classes of $6 \cdot \mathrm{Suz}$ and $6 \cdot \mathrm{Suz}: 2$}

We must correctly identify the conjugacy classes of $6 \cdot$ Suz:2 in order to reliably use the tables stored in GAP. In Section 5.1 we will also require the class fusion from 6.Suz to 6.Suz:2, and identifying the conjugacy classes of this subgroup is slightly harder (there are many pairs of algebraically conjugate classes).

We identify a conjugacy class by using class invariants such as element order, centraliser order, and character values, as in [21]. We obtain character values of the class representatives by taking the trace of various representing matrices. We can switch freely between representations by using words in standard generators [24] for the class representatives.

Our task is in some respects harder than that undertaken in [21] because we must consistently identify the algebraically conjugate classes. The various subtleties involved in doing this are described in [12]. (Our task is also somewhat easier since we are content using the 'straight line programs' (SLPs) produced by MAGMA rather than insisting on short SLPs.)

Let $a$ and $b$ be standard generators for Suz; then $a b$ is either in class $13 A$ or in $13 B$. If we choose which class $a b$ lies in, then from the Brauer character tables [13] we see that all of the other conjugacy class names of Suz and its covers now follow; that is, we have no more choice.

To identify the GAP conjugacy class names of 6 . Suz we begin in MAGma [23] with the 24-dimensional representation of $6 \cdot$ Suz:2 over $\mathbb{F}_{3}$ and use the web Atlas words to find a subgroup 6.Suz. MAGMA can produce a list of the conjugacy classes, with representatives, of both of these groups, and the class fusion.

We use the notation $\mathbf{d i}_{p}$ to refer to the $i$ th representation of (a cover of) Suz of dimension $d$ in characteristic $p$ with character printed in the Atlas [5] or Brauer table [13]. The ordering is as in the character tables, and $i \in\{a, b, c, \ldots\}$.

It is straightforward to identify most of the conjugacy classes of 6 . Suz from the element orders, centraliser orders, and complex character values computed from $\mathbf{1 2} \mathbf{a}_{0}$ (see [16]; John Bray has also described a characteristic zero representation of $6 . \mathrm{Suz}$ in twelve dimensions). We can be sure that we have the correct such representation (the one with character values printed in the Atlas) by using a word 
for the central element ' $-\omega$ ': In the complex representation it evaluates to the scalar matrix with $-\exp (2 \pi i / 3)$ on the diagonal. The representation $\mathbf{1 2}_{2}$ is over $\mathbb{F}_{4}$ and in MeatAxe notation we have $\mathbb{F}_{4}=\{0,1,2,3\}$ with 2 corresponding to $\omega$. Therefore in this representation our word must evaluate to the scalar matrix with 2 on the diagonal.

The conjugacy classes of 6 . Suz we cannot identify by this means are those lying above classes $12 D$ or $24 A$ of Suz (in each case there are two). The smallest dimension in which we can distinguish these classes is 1892 over $\mathbb{F}_{25}$ (a representation of $2 \cdot \mathrm{Suz}$ ). This representation is not available on the web Atlas so we must make it.

- From the 5-modular decomposition matrix for Suz we find that the representation we require is a constituent of the 5 -modular reduction of $4928 \mathbf{a}_{0}$.

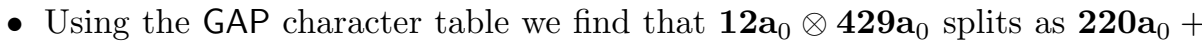
$4928 \mathrm{a}_{0}$.

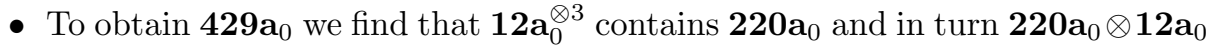
contains $429 \mathbf{a}_{0}$.

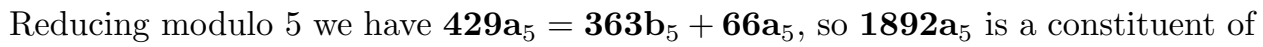
$\mathbf{3 6 3} \mathbf{b}_{5} \otimes \mathbf{1 2} \mathbf{a}_{5}$. As $12 \mathbf{a}_{5}$ is available on the web Atlas we can now construct $\mathbf{1 8 9 2 \mathbf { a } _ { 5 }}$ and identify the remaining conjugacy classes.

\section{Extending downwards: representations and the conjugacy classes}

We first use a 37 -dimensional representation of $3^{12}: 6$ Suz: 2 over $\mathbb{F}_{3}$, as constructed in [15], to determine the conjugacy classes. We then use a 38-dimensional representation of $3^{1+12}: 6$ Suz:2, again constructed in [15], to determine the conjugacy classes of $3^{1+12}: 6 \cdot$ Suz:2.

\subsection{A representation of $3^{12}: 6 \cdot$ Suz:2}

Let $V \cong 3^{12}$. Two matrices, $C_{2}$ and $D_{2}$ say, that are standard generators of 2.Suz:2 represented on $V$ are available on the web Atlas. There is a 24-dimensional representation of $6 \cdot \mathrm{Suz}: 2$ over $\mathbb{F}_{3}$, also available on the web Atlas, with (standard) generators $C_{6}$ and $D_{6}$. Hence via the projection map

$$
\text { 6.Suz: } 2 \rightarrow 2 \text { :Suz:2 given by } \quad C_{6} \mapsto C_{2} \text { and } D_{6} \mapsto D_{2}
$$

and the representation of $2 \cdot \operatorname{Suz}: 2$ on $V$ we obtain an un-faithful representation of 6. Suz:2 on $V$.

Let $g$ be a word in the two standard generators $C$ and $D$. Let $f_{6}(g)$ be $g$ evaluated in the 24-dimensional representation of 6 Suz: 2 and let $f_{2}(g)$ be the $12 \times 12$ matrix representing the projection down to $2 \cdot$ Suz:2. Then

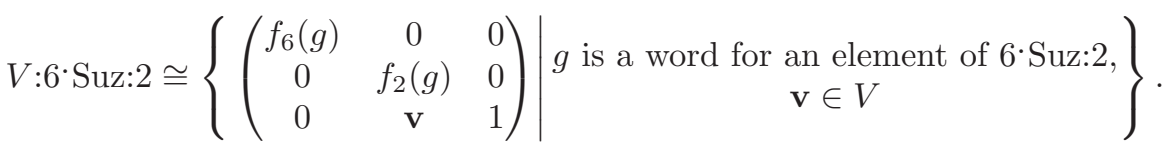

In practice we think of such an element as a pair $(g, \mathbf{v})$, where multiplication is given by $(g, \mathbf{v})(h, \mathbf{w})=(g h, \mathbf{v} h+\mathbf{w})$ and $\mathbf{v} h$ means the image of $\mathbf{v}$ under the action of $f_{2}(h)$. We determine the conjugacy classes of this group.

A conjugacy class $\left[f_{6}(g)\right]$ of 6 . Suz: 2 lifts to a number of conjugacy classes, say $\left[\left(g, \mathbf{v}_{i}\right)\right]$, of $V: 6 \cdot$ Suz:2 and the $\mathbf{v}_{i}$ are determined in two steps (cf.[19]). 
1. $V$ acts by conjugation on the coset $f_{2}(g) V$ by $\mathbf{w}:(g, \mathbf{v}) \mapsto(g,-\mathbf{w} g+\mathbf{v}+\mathbf{w})$.

2. These orbits are now fused by the action of the centraliser of $f_{6}(g)$. We must compute the centraliser in 6 Suz:2 of $f_{6}(\mathrm{~g})$ and use its image in $2 \cdot$ Suz:2 to determine the fusions. (The image in $2 \cdot$ Suz: 2 of $C_{6}$. Suz:2 $\left(f_{6}(g)\right)$ is not always equal to $C_{2} \cdot \operatorname{Suz}: 2\left(f_{2}(g)\right)$.)

All of these calculations are performed in Magma [23].

\subsection{A representation of $3^{1+12}: 6 \cdot$ Suz:2}

Elements of $3^{1+12}: 6$. Suz:2 can be thought of as triples $(g, \mathbf{v}, \lambda)$ where $(g, \mathbf{v})$ is as in Section 3.1 and $\lambda$ is in the new normal subgroup.

For convenience, write $g_{2}$ for $f_{2}(g)$ and $g_{6}$ for $f_{6}(g)$. We find a matrix $R$ corresponding to a symplectic form on $V$ that is invariant under 2 .Suz. Elements $g_{2}$ in 2 Suz:2 outside of this subgroup invert (that is, negate) the form. Equivalently, $R$ conjugates $g_{2}$ to its inverse transpose or to $-g_{2}^{-\top}$ (respectively) and so can readily be found using Standard Basis in the MeatAxe [14].

We now construct a 38 -dimensional representation of $3^{1+12}: 6$. Suz:2 as in [15] by taking a sub-direct product with 6 Suz:2. The matrices are of the form

$$
\left(\begin{array}{cccc}
g_{6} & 0 & 0 & 0 \\
0 & 1 & \mathbf{v} & \lambda \\
0 & 0 & g_{2} & -g_{2} R \mathbf{v}^{\top} \\
0 & 0 & 0 & \pm 1
\end{array}\right)
$$

where the sign is ' + ' if $g_{2}$ is in the inner half of $2 \cdot$ Suz:2, and ' - ' if it is outer. Standard generators for this group are defined in [15]: $C$ and $D$ are standard generators for the complementary 6 . Suz:2, and $E$ is a specified element of $3^{1+12}$ that is straightforward to find.

\subsection{Conjugacy classes of $3^{1+12}: 6 \cdot$ Suz:2}

We need to determine the conjugacy amongst the elements labelled $(g, \mathbf{v}, 0)$, $(g, \mathbf{v}, 1)$ and $(g, \mathbf{v}, 2)$ for each pair $(g, \mathbf{v})$ labelling a conjugacy class of $3^{12}: 6$ :Suz:2. Some of this work can be done directly in MAGMA, but we can speed things up considerably as follows.

We conjugate $(g, \mathbf{v}, \lambda)$ by $(h, \mathbf{w}, \mu)$ as $4 \times 4$ block matrices of the form given in equation (2), assuming that $g$ is inner. In the resulting matrix the $(2,3)$-entry is

$$
\mathbf{w}+\mathbf{v} h_{2}-\mathbf{w} h_{2}^{-1} g_{2} h_{2} .
$$

If $h$ is inner then the $(2,4)$-entry is the sum of $\lambda$ and

$$
-\mathbf{v} h_{2} R \mathbf{w}^{\top}+\mathbf{w} h_{2}^{-1} g_{2} h_{2} R \mathbf{w}^{\top}+\mathbf{w} h_{2}^{-1} g_{2} R \mathbf{v}^{\top}-\mathbf{w} R \mathbf{w} .
$$

If $h$ is outer then the $(2,4)$-entry is the sum of $-\lambda$ and

$$
-\mathbf{v} h_{2} R \mathbf{w}^{\top}+\mathbf{w} h_{2}^{-1} g_{2} h_{2} R \mathbf{w}^{\top}-\mathbf{w} h_{2}^{-1} g_{2} R \mathbf{v}^{\top}+\mathbf{w} R \mathbf{w} .
$$

Our task is to investigate those conjugates of $(g, \mathbf{v}, \lambda)$ by $(h, \mathbf{w}, \mu)$ that are of the form $\left(g, \mathbf{v}, \lambda^{\prime}\right)$; that is, what are the possible values of $\lambda^{\prime}$ ?

Clearly we require $h_{6}$ to centralise $g_{6}$, so $h_{2}$ centralises $g_{2}$. From (3) we require $\mathbf{v}-\mathbf{v} h_{2}=\mathbf{w}-\mathbf{w} g_{2}$, and we can use this condition as follows. Let $G$ be the image in $2 \cdot$ Suz:2 of the centraliser in 6.Suz:2 of $g_{6}\left(\right.$ so $\left.h_{2} \in G\right)$ and let $H$ be the stabiliser in 
$G$ of $\mathbf{v}$. Let $T$ be a transversal for $H$ in $G$. The dichotomy between $h_{2}$ being inner and outer forces us to use both an inner and outer representative for each coset: if $G$ contains an outer element $q$ then we let $T^{\prime}=\{t, q t \mid t \in T\}$. We now need only examine pairs $\left(h_{2}, \mathbf{w}\right)$ for $h_{2} \in T^{\prime}$ and $\mathbf{w}$ in the set

$$
\left\{\mathbf{w} \in V \mid \mathbf{w}-\mathbf{w} g_{2}=\mathbf{v}-\mathbf{v} h_{2}\right\} .
$$

For each of these pairs $\left(h_{2}, \mathbf{w}\right)$ we calculate $\lambda^{\prime}-\lambda$ according to equation (4) or equation (5), and we use this in the following case analysis. We also compute a fingerprint $L(g, \mathbf{v}, \lambda)$ which we use to prove non-fusion. Specifically, we store a list of randomly chosen polynomials $w_{1}, w_{2}, \ldots, w_{m}$ and given a matrix $X$ we produce a list $L(X)=\left(\operatorname{rk} w_{1}(X), \ldots\right.$, rk $\left.w_{m}(X)\right)$ of integers. If $L(X) \neq L(Y)$ then $X$ is not conjugate to $Y$.

1. If $L(g, \mathbf{v}, 0), L(g, \mathbf{v}, 1)$, and $L(g, \mathbf{v}, 2)$ are distinct then there can be no fusion.

2. If two of the fingerprints are distinct and $h$ is outer then the classes with equal fingerprints fuse.

3. If two of the fingerprints are distinct and $g$ is not centralised by any outer element then no fusion occurs.

4. Otherwise proceed as described to find non-zero values for formula (4) or formula (5).

(a) If $h$ is inner and formula (4) is non-zero then all three classes fuse.

(b) If $h$ is outer then a value in formula (5) of $\begin{array}{llll}0 \\ 1 \\ 2\end{array}$ fuses classes $\begin{array}{lll}1 & \& & 2 \\ 0 & \& & 1 \\ 0 & \& & 2\end{array}$.

Note that it is not necessarily the case that a value of 0 occurs, as we have not always chosen the zero vector as the representative of its orbit. If any two of these values occur, then all three classes fuse.

If $g$ is in the outer half of $6 \cdot \mathrm{Suz}: 2$, then all three pre-images are conjugate. After performing the appropriate $4 \times 4$ block matrix multiplications this is easy to see because the $(2,4)$-entry now contains $\mu$ as a summand.

We find there to be 533 conjugacy classes of $3^{1+12}: 6 \cdot$ Suz:2.

\section{The quotient $3^{1+12 \cdot} \cdot 2 \cdot \mathrm{Suz}: 2$}

We have been very careful to ensure that our generators $C, D$, and $E$ for the group $3^{1+12}: 6 \cdot$ Suz:2 are the same as those used in [15]. Therefore, given a word for an element in $3^{1+12}: 6$. Suz:2, we obtain its projection to $3^{1+12} \cdot 2 \cdot$ Suz:2 by simply evaluating the word in the corresponding generators.

However, there is still a problem. The group 6.Suz:2 has an outer automorphism that has the effect of multiplying elements in the outer half of the group by the central involution, $C^{2}$. Thus it may be possible that our standard generators $C, D$ and $E$ correspond to $C^{-1}, D$ and $E$ in [15].

Of course this problem is easily resolved, and fortunately it is easily detected. We found a word for an element of $3^{1+12}: 6 \cdot$ Suz: 2 that has order 2 and such that if we need to replace $C$ with $C^{-1}$ then in $3^{1+12} \cdot 2 \cdot$ Suz:2 the corresponding element has order 6 .

Another great benefit of consistency with [15] is that this work provides a word for an element, $q$ say, in the 3-group that we must quotient out. This is very 
important because there are two non-isomorphic groups of shape $3^{1+12} \cdot 2 \cdot$ Suz:2 and only one of them is isomorphic to $N(3 B)<\mathbb{M}$.

\subsection{Fusion to $\mathbb{M}$}

Once MAGMA has created a base and strong generating set for our 38-dimensional $\mathbb{F}_{3}$-representation of $\langle C, D, E\rangle=3^{1+12}: 6 \cdot$ Suz:2 it can quickly produce a word in these generators for a given element. Using our results from the previous section we paste together — as in the matrices (2) — a conjugacy class representative and then obtain a word for it.

Using [1] we can determine the conjugacy class in $\mathbb{M}$ of any element of the group $3^{1+12 \cdot} \cdot 2 \cdot$ Suz:2, up to algebraic conjugacy and except for elements of order 27 . We calculate the conjugacy class in $\mathbb{M}$ to which the image in $3^{1+12} \cdot 2 \cdot$ Suz:2 of each of our 533 class representatives of $3^{1+12}: 6$ Suz:2 fuses. We can now answer the question: 'Which classes of $\mathbb{M}$ intersect $N(3 B)$ non-trivially?' The answer is as follows.

$\begin{array}{cccccccccccc}1 \mathrm{~A} & 2 \mathrm{~A} & 2 \mathrm{~B} & 3 \mathrm{~A} & 3 \mathrm{~B} & 3 \mathrm{C} & 4 \mathrm{~A} & 4 \mathrm{~B} & 4 \mathrm{C} & 4 \mathrm{D} & 5 \mathrm{~A} & 5 \mathrm{~B} \\ 6 \mathrm{~A} & 6 \mathrm{~B} & 6 \mathrm{C} & 6 \mathrm{D} & 6 \mathrm{E} & 6 \mathrm{~F} & 7 \mathrm{~B} & 8 \mathrm{~A} & 8 \mathrm{~B} & 8 \mathrm{C} & 8 \mathrm{D} & 8 \mathrm{E} \\ 8 \mathrm{~F} & 9 \mathrm{~A} & 9 \mathrm{~B} & 10 \mathrm{~B} & 10 \mathrm{C} & 10 \mathrm{D} & 10 \mathrm{E} & 11 \mathrm{~A} & 12 \mathrm{~A} & 12 \mathrm{~B} & 12 \mathrm{C} & 12 \mathrm{D} \\ 12 \mathrm{E} & 12 \mathrm{~F} & 12 \mathrm{G} & 12 \mathrm{H} & 12 \mathrm{I} & 12 \mathrm{~J} & 13 \mathrm{~B} & 14 \mathrm{C} & 15 \mathrm{~A} & 15 \mathrm{~B} & 15 \mathrm{C} & 15 \mathrm{D} \\ 16 \mathrm{~A} & 18 \mathrm{~A} & 18 \mathrm{~B} & 18 \mathrm{C} & 18 \mathrm{D} & 18 \mathrm{E} & 20 \mathrm{C} & 20 \mathrm{D} & 20 \mathrm{E} & 20 \mathrm{~F} & 21 \mathrm{~B} & 21 \mathrm{D} \\ 22 \mathrm{~B} & 24 \mathrm{~A} & 24 \mathrm{~B} & 24 \mathrm{C} & 24 \mathrm{D} & 24 \mathrm{E} & 24 \mathrm{~F} & 24 \mathrm{G} & 24 \mathrm{H} & 24 \mathrm{I} & 24 \mathrm{~J} & 26 \mathrm{~B} \\ 27 \mathrm{~A} & 27 \mathrm{~B} & 28 \mathrm{D} & 30 \mathrm{~A} & 30 \mathrm{C} & 30 \mathrm{D} & 30 \mathrm{E} & 30 \mathrm{~F} & 30 \mathrm{G} & 33 \mathrm{~A} & 33 \mathrm{~B} & 36 \mathrm{~A} \\ 36 \mathrm{~B} & 36 \mathrm{C} & 36 \mathrm{D} & 39 \mathrm{C} & 39 \mathrm{D} & 40 \mathrm{C} & 40 \mathrm{D} & 42 \mathrm{~B} & 42 \mathrm{D} & 45 \mathrm{~A} & 48 \mathrm{~A} & 54 \mathrm{~A} \\ 56 \mathrm{~B} & 56 \mathrm{C} & 60 \mathrm{C} & 60 \mathrm{D} & 60 \mathrm{E} & 66 \mathrm{~B} & 78 \mathrm{~B} & 78 \mathrm{C} & 84 \mathrm{~B} & & & \end{array}$

\subsection{The quotient map}

If $z \in 3^{1+12}: 6$. Suz:2 then we may take the coset $z\langle q\rangle$ as a conjugacy class representative for $3^{1+12} \cdot 2 \cdot$ Suz:2. It is therefore necessary to determine the conjugacy classes in $3^{1+12}: 6 \cdot$ Suz: 2 of $z q$ and $z q^{2}$.

We could wait until we have the completed character table, identify the conjugacy class of $q$, and then deduce the character table of the quotient $N(3 B)$ by examining the characters that have $q$ in their kernel. However, we choose to compute the quotient map sooner since this will give us access — via the quotient, then fusion - to the character table of $\mathbb{M}$ which we wish to use to check our calculations before we continue.

We begin with a list $L$ of all 533 conjugacy class labels $(g, \mathbf{v}, \lambda)$ for class representatives $z \in 3^{1+12}: 6$. Suz:2. We determine the conjugacy class labels $\left(g^{\prime}, \mathbf{v}^{\prime}, \lambda^{\prime}\right)$ of $z q$ and $\left(g^{\prime \prime}, \mathbf{v}^{\prime \prime}, \lambda^{\prime \prime}\right)$ of $z q^{2}$. (The three classes need not be distinct.) Any of these labels different from $(g, \mathbf{v}, \lambda)$ we remove from $L$, and we continue. On termination, $L$ is a list of labels of conjugacy classes of $3^{1+12}: 6 \cdot$ Suz:2 that are in bijective correspondence with the conjugacy classes of $3^{1+12} \cdot 2 \cdot$ Suz:2.

To determine the label of the conjugacy class of $z q$ we begin with a list $K$ of all class labels, which we first reduce to a list of possible labels for the class of $z q$. To do this we remove those elements of $K$ for which the class representative $k$ does not meet the following criteria.

1. The images in $3^{1+12} \cdot 2 \cdot$ Suz: 2 of $k$ and $z q$ fuse to the same conjugacy class in $\mathbb{M}$.

2. $z q$ and $k$ have the same fingerprint. 
This considerably reduces the size of $K$ : just under half of the time $|K|=1$; otherwise $|K|=2$ is typical and the worst case is $|K|=6$. We use the IsConjugate function in MAgma to determine which class in $K$ contains $z q$. However, since a false result takes considerably longer than true we treat IsConjugate as a Las Vegas algorithm - we test all possibilities at once on our different machines, and the one that returns true finishes first.

This process must be repeated for $z q^{2}$.

\section{Characters of $3^{1+12}: 6 \cdot$ Suz:2 - easy cases}

Now that we have the conjugacy classes, power maps, and the projection map, all that remains is to find the 533 irreducible characters! There are 174 irreducible characters of 6 . Suz:2 which we inflate to $3^{1+12}: 6$. Suz:2. Finding the remaining 359 characters is somewhat harder, and is the subject of the remainder of this paper.

\subsection{Induction from $3^{1+12}: 6 \cdot \mathrm{Suz}$}

Let $\psi$ and $\bar{\psi}$ be the two degree-729 complex irreducible characters of $3^{1+12}$. They both extend to $G=3^{1+12}: 6$ : Suz. We need the values of $\psi$ and $\bar{\psi}$ on our class representatives that lie in $G$. We shall construct the representations affording modular reductions of $\psi$ and $\bar{\psi}$ over a field of characteristic coprime to $|G|$. We can then lift the eigenvalues of the representing matrices to $\mathbb{C}$ in order to determine the character values.

Specifically, we construct our representations over $\mathbb{F}_{31}$ by following the instructions in $[\mathbf{1 5}]$. For a class representative $g$ we use the Conway polynomial $[\mathbf{1 2}, \mathbf{1 7}]$ of degree $d$ over $\mathbb{F}_{31}$ to make a field extension, where $d$ is the least integer such that $\left(31^{d}-1\right) /|g| \in \mathbb{N}$. This extension contains the eigenvalues of $g$.

Let $\xi$ be a primitive element of the extension field, that is, a root of the Conway polynomial. Then

$$
\zeta_{d}=\xi^{\left(31^{d}-1\right) /|g|}
$$

is the canonical generator of a subgroup of the multiplicative group of the field that contains the eigenvalues of $g$.

In characteristic zero the eigenvalue $\zeta_{d}^{n}$ lifts to $\exp ((2 \pi i / d) n)$. The use of Conway polynomials ensures that our eigenvalues are lifted consistently. Thus we avoid having to extend the field so that it contains all eigenvalues of all elements of $G$; this would be too difficult, as such a field would be very large.

Let $\hat{\eta} \in \operatorname{Irr}(6 \cdot \mathrm{Suz})$ inflate to $\eta \in \operatorname{Irr} G$, so $\psi \eta \uparrow G: 2 \in \operatorname{Irr} G: 2$. Now, for $g \in G$,

$$
\begin{aligned}
\psi \eta \uparrow G: 2(g) & =\frac{1}{2|G|} \sum_{x \in G} \psi\left(g^{x}\right) \eta\left(g^{x}\right)+\frac{1}{2|G|} \sum_{x \in G: 2 \backslash G} \psi\left(g^{x}\right) \eta\left(g^{x}\right) \\
& =\frac{1}{2} \psi(g) \eta(g)+\frac{1}{2} \bar{\psi}(g) \bar{\eta}(g)
\end{aligned}
$$

and there are two cases to consider. Write

$$
\psi^{\prime}(g)= \begin{cases}\psi(g)+\bar{\psi}(g) & \text { if } g \text { is in the inner half of } G: 2 \\ 0 & \text { if } g \text { is in the outer half of } G: 2\end{cases}
$$

1. In the splitting case $\eta$ extends in two ways, giving characters $\eta^{+}$and $\eta^{-}$say, which are equal to $\eta$ on inner elements. The characters do not vanish on all 
outer elements simultaneously, and for outer $h$ we have $\eta^{+}(h)=-\eta^{-}(h)$. Thus equation (6) gives $\psi \eta \uparrow G: 2=\psi^{\prime} \eta^{ \pm}$(it is zero on outer elements, so both choices of sign give the same character). We thus obtain

$$
\begin{aligned}
\left\langle\psi^{\prime} \eta^{ \pm}, \psi^{\prime} \eta^{ \pm}\right\rangle_{G: 2} & =\frac{1}{2|G|} \sum_{g \in G} \psi^{\prime}(g) \eta^{ \pm}(g) \overline{\psi^{\prime} \eta^{ \pm}(g)}+0 \\
& =\frac{1}{2}\langle(\psi+\bar{\psi}) \eta,(\psi+\bar{\psi}) \eta\rangle_{G} \\
& =1
\end{aligned}
$$

so the character $\psi^{\prime} \eta^{ \pm}$is irreducible. We obtain 46 irreducible characters in this way.

2. In the fusion case we obtain a single new character $\eta^{\prime}$ which takes values $\eta(g)+\bar{\eta}(g)$ if $g$ is inner and $\eta^{\prime}(g)=0$ if $g$ is outer. Hence

$$
\left\langle\psi^{\prime} \eta^{\prime}, \psi^{\prime} \eta^{\prime}\right\rangle_{G: 2}=\frac{1}{2}\langle(\psi+\bar{\psi})(\eta+\bar{\eta}),(\psi+\bar{\psi})(\eta+\bar{\eta})\rangle_{G}=2
$$

so $\psi^{\prime} \eta^{\prime}$ is not irreducible. From equation (6) it is clear to see that the irreducible constituents are $\psi \eta \uparrow G: 2(g)=\psi \eta(g)+\overline{\psi \eta}(g)$ and $\bar{\psi} \eta \uparrow G: 2(g)=$ $\bar{\psi} \eta(g)+\psi \bar{\eta}(g)$ ( $g$ inner, both are zero on outer elements). The values of these characters can be evaluated as we have identified the conjugacy classes of $6 \cdot$ Suz and the fusion map to 6 -Suz:2. This gives us 164 more irreducible characters.

\section{Characters of $3^{1+12}: 6 \cdot$ Suz:2 - hard cases}

Let $H<G=3^{1+12}: 6$ Suz:2 be one of the inertia groups, let $T$ be a transversal for $H$ in $G$, and let $\chi \in \operatorname{Irr} H$. Let $\pi$ be the permutation representation of $G$ on the cosets of $H$. Each $t_{i} \in T$ corresponds to a coset $t_{i} H$ which in turn corresponds to a point $i$. Now, $t_{i} g t_{i}^{-1} \in H$ if and only if $\pi\left(t_{i} g t_{i}^{-1}\right)$ fixes 1 which means that $\pi(g)$ fixes $i$. Hence

$$
\chi \uparrow G(g)=\sum_{i \in \operatorname{Fix} \pi(g)} \chi\left(t_{i} g t_{i}^{-1}\right) .
$$

If $H<K<G$ then $\chi \uparrow K \uparrow G=\chi \uparrow G$. We find it more convenient to work with a group $K$ in which $H$ has index 2 .

The characters of $H$ that we must induce to $G$ are products $\psi \eta$ where $\psi$ extends a suitable linear character of $3^{1+12}$ and $\eta$ is inflated from $\bar{H}=H / 3^{1+12}$. We let $K=\left\langle H, c_{2}\right\rangle$ where $c_{2}$ is the central involution of $6 \cdot \mathrm{Suz}: 2$ which fuses such characters in pairs $(\eta, \bar{\eta})$. Thus our strategy is as follows.

1. Identify the conjugacy classes of $\bar{H}$.

2. Obtain the permutation representation $\pi$ on the cosets of $K$.

3. For each $g$ and $i \in \operatorname{Fix} \pi(g)$ determine the conjugacy class in $\bar{H}$ of the image of $t_{i} g t_{i}^{-1}$. This will allow us to read off the values of $\eta$ from the character table of $\bar{H}$.

4. Evaluate $\psi$ explicitly.

5. Assemble the induced characters.

We have two cases of group $K$ to consider. One has has shape $3^{1+12}:(2 \times(3 \times$ $\left.\left.\mathrm{U}_{5}(2)\right): 2\right)$ and the other has shape $3^{1+12}:\left(2 \times 3 \cdot 3^{5}\left(\mathrm{M}_{11} \times 2\right)\right)$. We shall explain the 
procedure for the former case in detail; the procedure for the latter is similar, and therefore in section 6.5 it is only summarised.

\subsection{Obtaining the permutation representation}

We have a 13 -dimensional module over $\mathbb{F}_{3}$ for $2 \cdot$ Suz: 2 with matrices of the form

$$
\left(\begin{array}{cc}
f_{2}(g) & 0 \\
\mathbf{v} & 1
\end{array}\right)
$$

cf. equation (1). Let $\mathbf{e} \in 3^{12}$ be a vector fixed by $\mathrm{U}_{5}(2)<2 \cdot$ Suz:2. The space $\langle\mathbf{e}\rangle$ has 32760 images under the action of $2 \cdot$ Suz:2 on the quotient 12-dimensional module. In the 13 -dimensional module there are $3 \times 32760=98280$ images of $\langle(\mathbf{e}, 0)\rangle$. To see this, recall the matrix $R$ that represents the invariant symplectic form, and let $\mathbf{v} \in \mathbb{F}_{3}^{12}$ have $\mathbf{v} R \mathbf{e}^{\top} \neq 0$. The $13 \times 13$ matrix

$$
\left(\begin{array}{cc}
I_{12} & -R \mathbf{v}^{\top} \\
\mathbf{v} & 1
\end{array}\right) \in 3^{12}: 2 \cdot \mathrm{Suz}: 2
$$

thus has orbit $\{(\mathbf{e}, 0),(\mathbf{e}, 1),(\mathbf{e},-1)\}$ on the 13-dimensional module. Therefore the stabiliser in $3^{12}$ of $\langle(\mathbf{e}, 0)\rangle$ is precisely the set of those matrices of the form given above but for which $\mathbf{v} \in\langle(\mathbf{e}, 0)\rangle^{\perp}$ (with respect to the bilinear form $R$ ) and so the full stabiliser is $3^{11}:\left(2 \times \mathrm{U}_{5}(2): 2\right)$.

Using the MEATAxE [14] vector permute program (zvp -p) we obtain permutations $\hat{C}, \hat{D}$ and $\hat{E}$ for the actions of $C_{13}, D_{13}$ and $E_{13}$ on 98280 points. This is a representation of $3^{12}: 2 \cdot$ Suz: 2 .

There is a representation of $6 \cdot \mathrm{Suz}: 2$ on 5346 points available on the web Atlas on standard generators $\tilde{C}$ and $\tilde{D}$, say. We re-number the 5346 points as 98281 to 103626 and make permutations

$$
C=\hat{C} \tilde{C}, \quad D=\hat{D} \tilde{D}, \quad \text { and } \quad E=\hat{E} \tilde{E} \quad\left(\text { where } \tilde{E}=\operatorname{Id}_{\langle\tilde{C}, \tilde{D}\rangle}\right)
$$

on 103626 points representing $G=3^{12}: 6 \cdot$ Suz:2. The stabiliser of point 1 becomes $3^{11}:\left(2 \times\left(3 \times \mathrm{U}_{5}(2)\right): 2\right)$ which is extended to $K=3^{12}:\left(2 \times\left(3 \times \mathrm{U}_{5}(2)\right): 2\right)$ by $E^{D}$.

We can obtain the 32760 point representation, $\pi$, of $3^{12}: 6 \cdot$ Suz:2 on the cosets of $K$ as follows ( $\pi$ represents faithfully the quotient Suz:2). The orbits of $E^{D}$ are blocks of imprimitivity in the 98280 point representation and fix all points in the 5346 point representation. An element of $2 \cdot$ Suz: 2 acts as \pm 1 on the 13 th co-ordinate, so the non-trivial blocks are preserved by $C$ and $D$ too. The resulting representation $\pi$ on 32760 points is both the action on the blocks and the action on the cosets of $K$.

\subsection{The inertia group and a quotient of it}

The inertia factor group is of course $\left(3 \times \mathrm{U}_{5}(2)\right): 2$ and we would like to lift a representation of this to the full inertia group, tensor it with an irreducible representation of $3^{12}$ fixed by and extended to the inertia group, and then induce to $G$.

But induction is transitive and the inertia group is a subgroup of index 2 of $K$ for which we have the representation on cosets, $\pi$. We may write

$$
\left.\left.K=3^{11} \cdot\left(\mathrm{S}_{3} \times\left(3 \times \mathrm{U}_{5}(2)\right): 2\right)\right) \quad \text { and } \quad \bar{K}=\mathrm{S}_{3} \times\left(3 \times \mathrm{U}_{5}(2)\right): 2\right) .
$$


(Recall that the 12 -dimensional $\mathbb{F}_{3}$-module for 6 -Suz:2 splits up as $1+10+1$ (uniserial) when restricted to $2 \times\left(3 \times \mathrm{U}_{5}(2)\right): 2$. The submodule $3^{11}$ is that exhibited in the shape of $K$ above.) We write

$$
\bar{K}=\left\langle e^{\prime}, c_{2}, c_{3}, u_{1}, u_{2}\right\rangle
$$

where $u_{1}$ and $u_{2}$ are standard generators for $\mathrm{U}_{5}(2): 2, e^{\prime}$ is the image of $E^{D}$, and $c_{2}$ and $c_{3}$ are elements in the centre of 6 . Suz of orders 2 and 3 respectively.

\subsection{Characters of $K$}

Observe that

$$
\mathrm{S}_{3}=\left\langle\bar{s}_{3}, \bar{s}_{2}\right\rangle \quad \text { where } \bar{s}_{3}=\left(\begin{array}{cr}
0 & 1 \\
-1 & -1
\end{array}\right) \text { and } \bar{s}_{2}=\left(\begin{array}{ll}
0 & 1 \\
1 & 0
\end{array}\right)
$$

over any field. The representations of $K$ that we are interested in have the form $\rho_{1} \otimes \rho_{3}$ or $\rho=\rho_{1} \otimes \rho_{2} \otimes \rho_{3}$ where the representations $\rho_{i}$ are defined by their images on the generators in equation (7) as given in the following table.

\begin{tabular}{c|ccccc} 
& $e^{\prime}$ & $c_{2}$ & $c_{3}$ & $u_{1}$ & $u_{2}$ \\
\hline$\rho_{1}$ & $I_{n}$ & $I_{n}$ & $I_{n}$ & $\bar{u}_{1}$ & $\bar{u}_{2}$ \\
$\rho_{2}$ & $I_{2}$ & $I_{2}$ & $\bar{s}_{3}$ & $\bar{s}_{2}$ & $\bar{s}_{2}$ \\
$\rho_{3}$ & $\bar{s}_{3}$ & $\bar{s}_{2}$ & $I_{2}$ & $I_{2}$ & $I_{2}$
\end{tabular}

(Implicitly, we have chosen a field and a representation $\rho_{1}$ of $\mathrm{U}_{5}(2): 2$.)

Note that the inclusion of $c_{2}$ in $K$ fuses the irreducible representations of $3^{12}$ in pairs. This is why we have a two-dimensional representation, $\rho_{3}$, for $\left\langle e^{\prime}, c_{2}\right\rangle$ instead of a one-dimensional representation for $\left\langle e^{\prime}\right\rangle$.

However, $\rho$ is not necessarily irreducible. If $\rho_{1}$ splits into two irreducibles when restricted to $\mathrm{U}_{5}(2)$, then we write $\rho_{1}=\tau+\bar{\tau}$ as representations for $\mathrm{U}_{5}(2)$, and write $\rho_{2}=\sigma+\bar{\sigma}$ where $\sigma$ and $\bar{\sigma}$ are irreducible representations for $\left\langle\bar{s}_{3}\right\rangle$. As representations for $3 \times \mathrm{U}_{5}(2)$ we have

$$
(\tau+\bar{\tau}) \otimes(\sigma+\bar{\sigma})=(\tau \otimes \sigma+\bar{\tau} \otimes \bar{\sigma})+(\tau \otimes \bar{\sigma}+\bar{\tau} \otimes \sigma)
$$

where the brackets on the right-hand side indicate the fusion in $\left(3 \times \mathrm{U}_{5}(2)\right): 2$. In particular, $\rho_{1} \otimes \rho_{2}$ is reducible.

\subsection{The induction}

For each of the 533 class representatives $g \in 3^{1+12}: 6 \cdot$ Suz:2 and for each $i \in$ Fix $\pi(g)$, we record the following information.

1. The conjugacy class in $\mathrm{U}_{5}(2): 2$ of $t_{i} g t_{i}^{-1}$ if it is in the outer half of the group. If it is in the inner half we must record the conjugacy class in $\mathrm{U}_{5}(2)$ in order to use equation (8).

2. $\xi_{2}(g, i)$ where

$$
\xi_{2}(g, i)= \begin{cases}1 & \text { if } \rho_{2}\left(t_{i} g t_{i}^{-1}\right) \text { is the identity; } \\ \exp \left(\frac{2 \pi i}{3}\right) & \text { if } \rho_{2}\left(t_{i} g t_{i}^{-1}\right)=\bar{s}_{3} \\ \exp \left(-\frac{2 \pi i}{3}\right) & \text { if } \rho_{2}\left(t_{i} g t_{i}^{-1}\right)=\bar{s}_{3}^{-1} \\ 0 & \text { otherwise. }\end{cases}
$$


When $\rho_{2}\left(t_{i} g t_{i}^{-1}\right)$ has order 3 , equation (8) requires us to know whether we have $\bar{s}_{3}$ or $\bar{s}_{3}^{-1}$; the trace alone is insufficient, so $\xi_{2}(g, i)$ records the information that we need. Note that the trace of $\rho_{2}\left(t_{i} g t_{i}^{-1}\right)$ is $\xi_{2}(g, i)+\overline{\xi_{2}(g, i)}$.

3. The trace of the matrix $\rho_{3}\left(t_{i} g t_{i}^{-1}\right), \xi_{3}(g, i)$ say.

These data can now be used to assemble 90 more irreducible characters.

- $\mathrm{U}_{5}(2): 2$ has 43 irreducible characters $\psi_{j}$. These give us 43 irreducible characters with values $\chi_{j}(g)=\sum_{i \in \mathrm{Fix} \pi(g)} \psi_{j}\left(t_{i} g t_{i}^{-1}\right) \xi_{3}(g, i)$.

- $\mathrm{U}_{5}(2)$ has 13 characters that extend to characters $\psi_{j} \in \operatorname{Irr} \mathrm{U}_{5}(2): 2$. From these we obtain 13 more irreducible characters with values

$$
\chi_{j}(g)=\sum_{i \in \operatorname{Fix} \pi(g)} \psi_{j}\left(t_{i} g t_{i}^{-1}\right)\left(\xi_{2}(g, i)+\overline{\xi_{2}(g, i)}\right) \xi_{3}(g, i) .
$$

- $\mathrm{U}_{5}(2)$ has 17 pairs of characters $\left(\psi_{j}, \bar{\psi}_{j}\right)$ that fuse in $\mathrm{U}_{5}(2): 2$. We obtain 34 more irreducible characters with values given by

$$
\chi_{j}(g)=\sum_{i \in \operatorname{Fix} \pi(g)}\left(\psi_{j}\left(t_{i} g t_{i}^{-1}\right) \xi_{2}(g, i)+\bar{\psi}_{j}\left(t_{i} g t_{i}^{-1}\right) \overline{\xi_{2}(g, i)}\right) \xi_{3}(g, i)
$$

and

$$
\bar{\chi}_{j}(g)=\sum_{i \in \operatorname{Fix} \pi(g)}\left(\psi_{j}\left(t_{i} g t_{i}^{-1}\right) \overline{\xi_{2}(g, i)}+\bar{\psi}_{j}\left(t_{i} g t_{i}^{-1}\right) \xi_{2}(g, i)\right) \xi_{3}(g, i) .
$$

\subsection{The other inertia group}

Here we consider the inertia group $H=3^{12}:\left(3 \cdot 3^{5}:\left(\mathrm{M}_{11} \times 2\right)\right)$ with quotient $\bar{H}=$ $3 \times 3 \cdot 3^{5}:\left(\mathrm{M}_{11} \times 2\right)$. These groups have index 2 in

$$
K=3^{12}:\left(2 \times 3 \cdot 3^{5}:\left(\mathrm{M}_{11} \times 2\right)\right) \quad \text { and } \quad \bar{K}=\mathrm{S}_{3} \times 3 \cdot 3^{5}:\left(\mathrm{M}_{11} \times 2\right)
$$

respectively, and $K$ has index 232960 in $3^{12}: 6 \cdot$ Suz:2.

To make the permutation representation $\pi$, we locate $3^{5}:\left(\mathrm{M}_{11} \times 2\right)<2$ Suz:2 using words from the web Atlas. As before, we spin up a fixed vector, this time obtaining permutations on 968880 points. Again, the action on the 232960 blocks of imprimitivity gives us the required representation $\pi$.

We now have $\rho_{1}$ being a representation of $3 \cdot 3^{5}:\left(\mathrm{M}_{11} \times 2\right)$. This already represents the central 3-elements of $6 \cdot \mathrm{Suz}$, and therefore we do not need $\rho_{2}$. The representation $\rho_{3}$ of $\mathrm{S}_{3}$ is as before.

The group $3 \cdot 3^{5}:\left(\mathrm{M}_{11} \times 2\right)$ is relatively small and easy to work in, for example obtained as a subgroup in the 5346-point permutation representation of 6.Suz:2. This will be our representation $\rho_{1}$. Using this, GAP can compute the character table, so we store it together with explicit class representatives $x_{j}(1 \leqslant j \leqslant 59)$. In MAGMA we can use the IsConjugate function to find $j_{(g, i)}$ such that $\rho_{1}\left(t_{i} g t_{i}^{-1}\right)$ is conjugate to $x_{j}$ in $3 \cdot 3^{5}:\left(\mathrm{M}_{11} \times 2\right)$.

Let $g \in 3^{1+12}: 6 \cdot$ Suz:2 be one of the 533 class representatives. For each $i \in$ Fix $\pi(g)$ we record:

1. the label $j_{(g, i)}$;

2. the trace $\xi(g, i)$ of $\rho_{3}\left(t_{i} g t_{i}^{-1}\right)$. 
For each of the 59 characters $\psi_{k}$ of $3 \cdot 3^{5}:\left(\mathrm{M}_{11} \times 2\right)$ we obtain $\chi_{k} \in \operatorname{Irr}\left(3^{1+12}: 6 \cdot \operatorname{Suz}: 2\right)$ with values

$$
\chi_{k}(g)=\sum_{i \in \operatorname{Fix} \pi(g)} \psi_{k}\left(x_{j_{(g, i)}}\right) \xi(g, i) .
$$

This completes the table of irreducible characters.

\section{Character tables}

With all the data we have computed to hand, we assemble first the character table of $3^{1+12}: 6$ Suz:2. We then use the quotient map from Section 4.2 to assemble the character table of the maximal subgroup of the Monster of shape $3^{1+12} \cdot 2 \cdot$ Suz:2. Because of the way we have labelled the conjugacy classes, it is also easy to produce the character table for the quotient $3^{12}: 6$ Suz:2.

\subsection{Power maps}

Let $z$ be a class representative and $p \in\{2,3,5,7,11,13\}$. For each $p$ we must determine the conjugacy class of $z^{p}$. The following information allows us to produce an approximate $p$ th power map, that is, a list of possible conjugacy classes for $z^{p}$ :

1. power maps lifted from $6 \cdot \mathrm{Suz}: 2$;

2. power maps of $\mathbb{M}$ restricted to $N(3 B)$ and lifted using the projection map calculated in Section 4.2;

3. fingerprints;

4. the power maps construction functions in GAP. Some of these make use of the characters.

This produces a unique power map in all cases except $p=3$, where there are $2^{57} 3^{18}$ possible power maps. Testing these with MAGMA is feasible using the same technique we used to find the quotient map in Section 4.2. As in Section 4.2 the explicit calculation serves as a check on our results — of the $2^{57} 3^{18}$ possible power maps, we reduce to a unique power map.

\subsection{The finished tables}

The character tables are available for download from Appendix A, or from the first author's website, http://www.maths.qmul.ac.uk/ ${ }^{\sim}$ rwb/table. They will be included in the next version of the GAP character table library [3]. We have the following connections between character tables:

- quotient map from $3^{1+12}: 6 \cdot$ Suz:2 to $3^{12}: 6 \cdot$ Suz:2;

- quotient map from $3^{1+12}: 6 \cdot \mathrm{Suz}: 2$ to $3^{1+12} \cdot 2 \cdot \mathrm{Suz}: 2$;

- quotient map from $3^{1+12}: 6 \cdot$ Suz:2 to $6 \cdot$ Suz:2;

- fusion map from $6 \cdot \mathrm{Suz}: 2$ to $3^{1+12}: 6 \cdot \mathrm{Suz}: 2$;

- fusion map from $3^{1+12 \cdot} \cdot 2 \cdot$ Suz:2 to $\mathbb{M}$. Because we now have the full character tables available we can determine the class fusion completely, an improvement over [1] where classes could only be distinguished up to algebraic conjugacy and $27 A$ could not be distinguished from $27 B$. Algebraically conjugate classes that do not intersect $N(3 B)$, however, remain without explicit representatives. 
These various connections allow us to test our tables against known data. For example, we have checked that for all $\chi \in \operatorname{Irr} \mathbb{M}$ we have $\langle\chi \downarrow N(3 B), \phi\rangle \in \mathbb{N} \cup\{0\}$ for all $\phi \in \operatorname{Irr} N(3 B)$. However, the main checks on our calculations have been ongoing throughout: besides keeping them consistent with existing character tables through our explicit computations, we have ensured that we are consistent with representations of the groups that we have been using, and with previous work on the groups.

\section{Conclusions}

We have computed the character tables of the maximal subgroup $N(3 B)$ of the Monster and of two other groups. Importantly, we have also determined the class fusion from $N(3 B)$ to $\mathbb{M}$. Our careful identification of the conjugacy classes means that our tables are suitable for inclusion in GAP and so are of general use.

Finally, it seems that certain recent advances have brought the computation of the character table of $N(3 B)$ into the realm of the 'compute on demand' approach. In particular, we note the following.

1. A representation of $N(3 B)$ over $\mathbb{F}_{3}$ in 72 dimensions [2] is now available on the web Atlas.

2. A new approach has been developed to computing in matrix groups [20]. The author of this software checked for us that $3^{1+12}: 2 \cdot$ Suz:2 has 533 conjugacy classes, and that $N(3 B)$ has 253 classes. This software will be included in MAGMA.

3. A new character table algorithm [22] is now included in Magma.

Some details of both [20] and [22] are available at

$$
\text { http://www . maths . warwick.ac.uk/ dfh/magma05.html. }
$$

Using the representation (1), the software (2) can produce conjugacy class representatives, and effectively test elements for conjugacy. These are pre-requisites for algorithm (3), which is also a considerable improvement over the traditional Dixon-Schneider method.

\section{Appendix A. The character tables}

The tables referred to in Section 7.2 can be found at

$$
\text { http://www. Ims.ac.uk/jcm/10/lms2006-016/app-a. }
$$

\section{References}

1. R. W. Barraclough and R. A. Wilson, 'Conjugacy class representatives for the Monster group', LMS J. Comput. Math. 8 (2005) 205-216, http://www.lms.ac.uk/jcm/8/lms2005-007.161, 166, 172

2. J. N. BRAY and R. A. Wilson, 'Explicit construction of maximal subgroups of the Monster', J. Algebra, to appear. 173 
3. Thomas Breuer, Manual for the GAP character table library, Version 1.1 (Lehrstuhl D für Mathematik, Rheinisch Westfälische Technische Hochschule, Aachen, Germany, 2004). 161, 172

4. J. H. Conway, 'A simple construction for the Fischer-Griess monster group', Invent. Math. 79 (1985) 513-540. 161

5. J. H. Conway, R. T. Curtis, S. P. Norton, R. A. Parker and R. A. WILSON, with computational assistance from J. G. Thackray, An ATLAS of finite groups. Maximal subgroups and ordinary characters for simple groups (Oxford University Press, Eynsham, 1985). 162

6. The GAP Group, 'GAP - groups, algorithms, and programming', Version 4.3 (2002), http://www.gap-system.org. 161

7. R. L. Griess, 'The friendly giant', Invent. Math. 69 (1982) 1-102. 161

8. P. E. Holmes and R. A. Wilson, 'A new maximal subgroup of the Monster', J. Algebra 251 (2002) 435-447. 161

9. P. E. Holmes and R. A. Wilson, 'A computer construction of the monster using 2-local subgroups', J. London Math. Soc. 67 (2003) 349-364. 161

10. P. E. Holmes and R. A. Wilson, 'PSL 2 (59) is a subgroup of the Monster', J. London Math. Soc. (2) 69 (2004) 141-152. 161

11. I. M. IsAacs, Character theory of finite groups (Dover, 1994). 161

12. C. Jansen, K. Lux, R. A. Parker and R. A. Wilson, An Atlas of Brauer characters, London Mathematical Society Monographs (N.S.) 11 (Oxford University Press, 1995). 162, 167

13. C. JAnsen and J. Müller, 'The 3-modular decomposition numbers of the sporadic simple Suzuki group', Comm. Algebra 25 (1997) 2437-2458. 162

14. Lehrstuhl D für Mathematik, RWTH Aachen, 'The MeatAxe - computing with modular representations', 2004, http://www.math.rwth-aachen.de/homes/MTX/. 164, 169

15. S. A. Linton, R. A. Parker, P. Walsh and R. A. Wilson, 'Computer construction of the monster', J. Group Theory 1 (1998) 307-337. 161, 162, $163,164,165,167$

16. F. LüBECK et al., 'The modular atlas', 2004, http://www.math.rwth-aachen.de/ 'MOC. 162

17. F. LüBECK, 'Conway polynomials for finite fields', 2004, http://www.math.rwth-aachen.de:8001/ ${ }^{\sim}$ Frank. Luebeck/data/ConwayPol. 167

18. S. P. Norton, 'Counting nets in the Monster', Combinatorics and geometry, Durham, 2001 (ed. A. A. Ivanov, M. W. Liebeck and J. Saxl, World Science Publishing Company, 2003) 227-232. 161

19. A. Seress, Permutation group algorithms (Cambridge University Press, 2003). 163

20. M. Stather, 'Computing in matrix groups', Seminars at 'Groups St Andrews 2005' and Magma Workshop on Group Theory and Algebraic Geometry, Warwick, 2005; see http://www.maths.warwick.ac.uk/ dfh/magma05.html. 173 
21. I. A. I. Suleiman, P. G. Walsh and R. A. Wilson, 'Conjugacy classes in sporadic simple groups', Comm. Algebra 28 (2000) 3209-3222. 162

22. W. Unger, 'Computing the character table of a finite group', J. Symbolic Comput. 41 (2006) 847-862. 173

23. University of Sydney, 'The Magma Handbook', 2004, http://magma.maths .usyd.edu.au/magma/htmlhelp/MAGMA.htm. 162, 164

24. R. A. WiLson, 'Standard generators for sporadic simple groups', J. Algebra 184 (1996) 505-515. 162

R. W. Barraclough R.W.Barraclough@qmul.ac.uk

http://www . maths. qmul.ac.uk/ ${ }^{\sim}$ rwb

R. A. Wilson R.A.Wilson@qmul.ac.uk

School of Mathematical Sciences

Queen Mary, University of London

Mile End Road

London E1 4NS

United Kingdom 